\title{
REFERENCES
}

1. R. P. Boas, Jr., Entire functions, New York, 1954.

2. R. C. Buck, A class of entire functions, Duke Math. J. vol. 13, pp. 541-559.

3. L. A. Rubel, Necessary and sufficient conditions for Carlson's theorem on entire functions, Proc. Nat. Acad. Sci. U.S.A. vol. 41, pp. 601-603.

UNIVERSITY OF WISCONSIN AND

CorNell University

\section{ON COMMUTATORS AND JACOBI MATRICES ${ }^{1}$}

\author{
C. R. PUTNAM
}

1. All operators in this paper are bounded linear transformations on a Hilbert space. The commutator $C$ of two operators $A$ and $B$ is defined by

$$
C=A B-B A .
$$

The closure, $W$, of the set of values $(C x, x)$ when $\|x\|=1$ is a closed convex set (Hausdorff, cf. [8, p. 34]). A complex number $z$ will be said to belong to the interior of $W$ if $z$ is in $W$ and if one of the following conditions holds: (i) If $W$ is two-dimensional, then $z$ does not lie on the boundary of $W$; (ii) If $W$ is a line segment, then $z$ is not an end point; (iii) $W$ consists of $z$ alone.

It was shown in [4] that if $A$ (or $B$ ) is normal, or even semi-normal, so that $A A^{*}-A^{*} A$ is semi-definite, then 0 belongs to $W$, but is not necessarily in the interior of $W$. (That, for arbitrary $A$ and $B$, in general 0 need not even belong to $W$ was shown in [2].) In fact, if $A=\left(a_{i j}\right)$ is defined by $a_{i, i+1}=1, a_{i j}=0$ if $j \neq i+1$, then $C=A A^{*}-A^{*} A$ $=\left(c_{i j}\right)$ is the self-adjoint matrix all elements of which are zero except $c_{11}=1$. Consequently, $C \geqq 0$ with a spectrum consisting of $\lambda=0,1$; hence $W$ is the segment $0 \leqq \lambda \leqq 1$ and 0 is not in the interior of $W$. Moreover, the above $C$ can also be expressed by $C=D A^{*}-A^{*} D$ where $D$ is the (self-adjoint) Jacobi matrix $A+A^{*}$; cf. $\S 5$ below.

The problem to be considered in the present paper is that of determining a sufficient condition guaranteeing that 0 belongs to the interior of $W$. Such a condition yields information concerning the spec-

Received by the editors November 25, 1955.

1 This work was supported by the National Science Foundation research grant NSF-G-481. 
trum of $C$, at least if $C$ is normal (since $W$ is then the least convex set containing the spectrum of $C$ ). Concerning the possible spectra for commutators (not necessarily normal) see [3, p. 198].

2. There will be proved the following principal

Lemma. Let $A$ be normal with the spectral resolution

$$
A=\int z d K(z)
$$

Let $S$ be an arbitrary measurable (here, and in the sequel, "measurable" implies "measurable with respect to $K(z)$ ") set in the complex plane with a covering $\left\{\Delta_{1}, \Delta_{2}, \Delta_{3}, \cdots\right\}$ by pair-wise disjoint measurable sets $\Delta_{n}$ of diameter $d_{n}$. Suppose that $H=C+C^{*}$ is non-negative definite with the self-adjoint square root $H^{1 / 2}$. Then, if $x$ is any element of Hilbert space, there holds the inequality

$$
\left\|H^{1 / 2} \int_{S} d K x\right\|^{2} \leqq 4\|B\|\|x\|^{2}\left(\sum_{n} d_{n}\right) .
$$

Moreover, if $A$ is self-adjoint or unitary, then

$$
\left\|H^{1 / 2} \int_{S} d K x\right\|^{2} \leqq 4\|B\|\|x\|^{2}(\text { meas } S),
$$

where the measure refers to the ordinary one-dimensional Lebesgue measure on a segment or a circle according as $A$ is self-adjoint or is unitary.

Proof of the lemma. Let $\Delta$ denote an arbitrary measurable set in the complex plane and let $\Delta K=\int_{\Delta} d K$. Multiplication by $\Delta K$ on the left and on the right of the equation (1) yields

$$
\begin{aligned}
\Delta K C \Delta K & =\int_{\Delta} z d K B \Delta K-\Delta K B \int_{\Delta} z d K \\
& =\int_{\Delta}\left(z-z_{0}\right) d K B \Delta K-\Delta K B \int_{\Delta}\left(z-z_{0}\right) d K,
\end{aligned}
$$

where $z_{0}$ is an arbitrary constant. (Cf. [1] and [5] for calculations of a somewhat similar nature.) It is clear that

$$
\begin{aligned}
(\Delta K x, C \Delta K x)= & \left(\Delta K x, \int_{\Delta}\left(z-z_{0}\right) d K B \Delta K x\right) \\
& -\left(\Delta K x, \Delta K B \int_{\Delta}\left(z-z_{0}\right) d K x\right) .
\end{aligned}
$$


If adjoints are taken in (5), a relation (6') similar to (6) exists for $C^{*}$. Addition of (6) and $\left(6^{\prime}\right)$ followed by a majorization leads to $(\Delta K x$, $H \Delta K x) \leqq 4\|B\|\|\Delta K x\|^{2} d$, where $d$ is the diameter of $\Delta$. Consequently,

$$
\left\|B^{1 / 2} \Delta K x\right\| \leqq 2\|B\|^{1 / 2}\|\Delta K x\| d^{1 / 2} \text {. }
$$

Next, by (7),

$$
\begin{aligned}
\left\|H^{1 / 2} \int_{S} d K x\right\| & \leqq \sum\left\|H^{1 / 2} \int_{S \Delta_{n}} d K x\right\| \\
& \leqq \sum 2\|B\| 1 / 2\left\|\int_{S \Delta_{n}} d K x\right\|\left(\operatorname{diam} S \Delta_{n}\right)^{1 / 2} .
\end{aligned}
$$

In view of the inequalities $\sum_{n}\left\|\int_{S \Delta_{n}} d K x\right\|^{2}=\int_{S} d\|K x\|^{2} \leqq\|x\|^{2}$ and diam $S \Delta_{n} \leqq d_{n}$, an application of the Schwarz inequality to (8) now yields the desired relation (3). Relation (4) is readily verified to be a consequence of (3) (if one chooses the $\Delta_{n}$ for instance to be rectangles) and the proof of the lemma is now complete.

3. As a consequence of the lemma, there will be derived in this section the following

THEorem. (i) Suppose that $A$ is normal with the spectral resolution (2) and that

$$
I=\int_{S} d K
$$

for a measurable set $S$. If $S$ can be covered by a sequence of pair-wise disjoint measurable sets $\Delta_{n}$ for which $\sum_{n}\left(\operatorname{diam} \Delta_{n}\right)$ can be made arbitarily small, then 0 belongs to the interior of the set $W$ associated with $C$ of (1).

(ii) Moreover, if $A$ is self-adjoint or unitary, and if there exists a set $S=Z$ of (one-dimensional) measure zero for which (9) holds, then 0 belongs to the interior of the set $W$.

Proof of the theorem. Suppose, if possible, that 0 is not in the interior of $W$. Then choose $\theta$ so that the set $W_{\theta}(=W \exp (i \theta))$ belonging to $C=A(B \exp (i \theta))-(B \exp (i \theta) A(=C \exp (i \theta))$ lies entirely in the half-plane $R(z) \geqq 0$. (Cf. [4, p. 129].) It is clear that $H_{\theta}=C_{\theta}+C_{\theta}^{*}$ $\geqq 0$ and that $\|B \exp (i \theta)\|=\|B\|$. Consequently an application of (3) of the lemma shows that $\left\|H_{\theta}^{1 / 2} x\right\|^{2} \leqq 4\|B\|\|x\|^{2}\left(\sum_{n} d_{n}\right)$. Hence, in view of the hypothesis, $\left\|H_{\theta}^{1 / 2} x\right\|=0$ for every $x$, that is, $H_{\theta}^{1 / 2}$ (hence $H_{\theta}$ ) is the zero operator. Therefore

$$
C \exp (i \theta)=-(C \exp (i \theta))^{*},
$$


thus $C \exp (i \theta+i \pi / 2)$ is self-adjoint. Since $W_{\theta+\pi / 2}$ is therefore a segment of the real axis, the set $W$ is a segment containing the origin. If however, 0 is not in the interior of this segment, there exists an angle $\phi$ for which the set $W_{\phi}$ belonging to $C \exp (i \phi)$ lies on the real axis with left end point at 0 . In view of relation (10), which now holds for $\theta=\phi$, and the fact that $C \exp (i \phi)$ is self-adjoint, it follows that $C \exp (i \phi)=0$, hence $C=0$, in contradiction to the assumption that 0 is not in the interior of $W$. This completes the proof of (i) of the theorem.

The proof of part (ii) of the theorem is similar if one notes that (4) now applies to the set $S=Z$ and meas $Z=0$.

4. Various corollaries of the above theorem will be collected in this section.

COROLlaRY 1. If $A$ (or $B$ ) is normal with a pure point spectrum then 0 is in the interior of the set $W$.

The proof is clear from part (i) of the theorem if it is noted that $S$ can be chosen to be a denumerable point set.

COROllary 2. If $A$ (or $B$ ) is self-adjoint or unitary with spectrum of (one-dimensional) measure zero then 0 is in the interior of $W$.

The proof follows from part (ii) of the theorem if it noted that (9) must hold when $S$ is the spectrum of $A$.

Corollary 3. If $C=A A^{*}-A^{*} A$ is semi-definite, then either (i) $C=0$ or (ii) if $A+A^{*}$ has the spectral resolution $\int \lambda d E(\lambda)$, then $\int_{\mathrm{z}} d E<I$ for every (one-dimensional) zero set $Z$ on the real axis.

It is clear that

$$
C=\left(A+A^{*}\right) A^{*}-A^{*}\left(A+A^{*}\right),
$$

so that $A+A^{*}$ can be identified with $A$ of the theorem. If now (ii) of Corollary 3 fails to hold, so that $\int_{\mathrm{z}} d E=I$ for some zero set $Z$, then part (ii) of the theorem implies that 0 belongs to the interior of $W$. Since however the end points of $W$ belong to the spectrum of the (semi-definite) operator $C$, then $W$ must reduce to the single point 0 . Therefore $C=0$; that is, (i) of Corollary 3 holds.

5. On the spectra of Jacobi matrices. That $C$ of Corollary 3 and (11) need not be zero was shown by the example in $\$ 1$ where $A+A^{*}$ $=D=\left(d_{i j}\right)$ is the Jacobi matrix with $d_{i, i+1}=d_{i+1, i}=1, d_{i j}=0$ otherwise. (For a discussion of this type of Jacobi matrix (i.e., one in which the diagonal elements are 0 ) see $[8$, p. 236]. 
More generally if $A=\left(a_{i j}\right)$ is defined by $a_{i, i+1}=b_{i}$ and $a_{i j}=0$ for $j \neq i+1$, then $A+A^{*}=D=\left(d_{i j}\right)$ is the self-adjoint matrix with $d_{i, i+1}=b_{i}, d_{i+1, i}=b_{i}^{*}$, and $d_{i j}=0$ otherwise. Moreover it is easily verified that $C=D A^{*}-A^{*} D$ is the diagonal matrix with diagonal elements $\left|b_{1}\right|^{2},\left|b_{2}\right|^{2}-\left|b_{1}\right|^{2},\left|b_{3}\right|^{2}-\left|b_{2}\right|^{2}, \cdots$. If the $b_{i}$ satisfy

$$
0<\left|b_{1}\right| \leqq\left|b_{2}\right| \leqq\left|b_{3}\right| \leqq \cdots,
$$

then $D$ is a Jacobi matrix and $C(\neq 0)$ is non-negative definite. Thus, case (ii) of Corollary 3 applies and if $D=\int \lambda d E(\lambda)$ then $\int_{\mathbf{z}} d E<I$ for every zero set $Z$ of the $\lambda$ axis. (In particular, when (12) holds, $D$ cannot have a pure point spectrum, nor can its spectrum be a zero set.)

\section{REFERENCES}

1. B. Fuglede, A commutativity theorem for normal operators, Proc. Nat. Acad. SciU.S.A. vol. 36 (1950) pp. 35-40.

2. P. R. Halmos, Commutators of operators, Amer. J. Math. vol. 74 (1952) pp. 237-240.

3. - Commutators of operators, II, ibid. vol. 76 (1954) pp. 191-198.

4. C. R. Putnam, On commutators of bounded matrices, ibid. vol. 73 (1951) pp. 127131.

5. - On normal operators in Hilbert space, ibid. vol. 73 (1951) pp. 357-362.

6. F. Rellich, Der Eindeutigkeitssatz für die Lösungen quantenmechanische Vertauschungsrelationen, Göttinger Nachrichten, 1946, pp. 107-115.

7. H. Wielandt, Ueber die Unbeschränkheit der Operatoren der Quantenmechanik, Math. Ann. vol. 121 (1949) p. 21.

8. A. Wintner, Spektraltheorie der unendlichen Matrizen, Leipzig, 1929.

9. - The unboundedness of quantum-mechanical matrices, Physical Review vol. 71 (1947) pp. 738-739.

Purdue University 